\title{
The role of exosomes in tumor immunity
}

\author{
Huayu Yang", Lejia Sun", Yilei Mao \\ Department of Liver Surgery, Peking Union Medical College (PUMC) Hospital, PUMC \& Chinese Academy of Medical Sciences, Beijing 100730, \\ China \\ \#These authors contributed equally to this article. \\ Correspondence to: Yilei Mao. Department of Liver Surgery Peking Union Medical College (PUMC) Hospital, PUMC \& Chinese Academy of \\ Medical Sciences 1\# Shuai-Fu-Yuan, Wang-Fu-Jing, Beijing 100730, China. Email: pumch-liver@hotmail.com.
}

Submitted Nov 05, 2018. Accepted for publication Nov 30, 2018.

doi: 10.21037/atm.2018.12.03

View this article at: http://dx.doi.org/10.21037/atm.2018.12.03

Exosomes are extracellular nanovesicles with a size of 30 $150 \mathrm{~nm}$ (in diameter) and they can be secreted by different kinds of cells under both physiological and pathological conditions. Exosomes have a typical lipid bilayer structure and comprise proteins, lipids, messenger RNA, non-coding RNA and DNA from the original cells. They can transfer the contents from donor to target cells and contribute to the intercellular communication. It is universally acknowledged that exosomes play a pivotal role in cancer, cardiovascular disease, inflammation, neurodegenerative diseases, and viral infections. Exosomes have been extensively studied, especially in the field of tumor (1). Tumor released exosomes can promote tumor growth and metastasis by transferring non-coding RNA or protein (2). Moreover, tumor exosome even can determine organotropic metastasis (3). There are different kinds of integrins on exosomes. The exosomal integrins $\alpha 6 \beta 4$ and $\alpha 6 \beta 1$ were linked to lung metastasis, while exosomal integrin $\alpha v \beta 5$ was associated with liver metastasis.

Immune escape is the key mechanism of tumor development and tumor immunity has accepted more attention. The increasing number of studies show that exosomes have a great influence on tumor immunity. Tumor cells can evade host immunologic surveillance partly because of poorly immunogenic. What's surprising is that tumor released exosomes can regulate tumor immunogenicity. Moroishi et al. (4) found that Hippo Pathway Kinases LATS1/2-null tumor cells can secrete nucleic-acidrich exosomes, and then the exosomes induced a type I interferon response via the toll-like receptors-MYD88/ TRIF pathway to improve tumor immunogenicity. The discovery is helpful to better understand the mechanisms of regulating tumor immunogenicity. Besides, exosomes contribute significantly to intercellular communication and reprogramming of the tumor immune microenvironment. It is found that $\mathrm{T}$ cell activity was obviously suppressed when co-culturing tumor-released exosomes and human $\mathrm{T}$ cells (5). Mirzaei et al. (6) found that circulating exosomes from glioblastoma patients carried more the extracellular matrix protein tenascin-C (TNC) than healthy individuals. TNC can inhibited $\mathrm{T}$ cell proliferation through interaction with $\alpha 5 \beta 1$ and $\alpha v \beta 6$ integrins on $\mathrm{T}$ lymphocytes. After depleting TNC, exosomes suppressed $\mathrm{T}$ cell responses to a significantly lesser extent than control.

Recently, Chen et al. (7) reported that there was programmed death-ligand 1 (PD-L1) on the surface of the exosomes released by glioblastoma and the PD-L1 on exosomes can indeed bind to programmed death 1 (PD-1) on $\mathrm{T}$ cells to inhibit $\mathrm{T}$ cell activation, which can be blocked by anti-PD-1 therapy. The amount of PD-L1 on these exosomes significantly increased after stimulating with interferon- $\gamma($ IFN- $\gamma)$. In patients with metastatic melanoma, the level of circulating exosomal PD-L1 was associated with that the level of IFN- $\gamma$. Immune checkpoints, especially PD-1/PD-L1, blockade brings a breakthrough for tumor therapy. However, it is not satisfactory due to low response rate (8). The findings discussed above reveal new immune immunosuppressive mechanism and are helpful to improve anti-PD-1/PD-L1 therapy. Other immune checkpoints may have similar mechanisms. The level of PD-L1 in tumor tissues is a good indicator for prognosis and immunity reaction. Similarly, the level of PD-L1 in exosomes may be a promising indicator because the latter is suitable for dynamic evaluation. Besides, the exosomal PD-L1 has 
potential in distinguishing the patients who have high response to anti-PD-1/PD-L1 therapy.

Besides CD8+ killer $\mathrm{T}$ cell, other types of tumorassociated immune cells, such as dendritic cells (DCs), macrophagocyte, regulatory $\mathrm{T}$ cells (Tregs) and myeloidderived suppressor cells (MDSCs) can be regulated by esosomes. Exosomes released by lung carcinoma and breast cancer can inhibit the maturation and migration of DCs and promote the immune suppression of DCs (9). Shen et al. (10) found that exosomes can educate DCs. The HSP72 and HSP105 on the tumor exosomes surface can induce IL-6 secretion of DCs in a TLR2- and TLR4dependent manner, which resulted in promoting tumor metastasis. Macrophages are the most abundant infiltrating tumors immune-related cells. They display diverse phenotypes and functions depending on microenvironment. Macrophages can be polarized into classically (M1) or alternatively (M2) activated cells. The M2 plays a role in anti-inflammatory response, angiogenesis, and promoting tumor growth and metastasis. Hypoxic exosomes can activate macrophages to the M2 phenotype in a HIF1a or HIF-2a-dependent manner. Mechanistically, the exosomes carrying miR-301a-3p and miR-301a$3 \mathrm{p}$ can induce the M2 polarization of macrophages by activating the PTEN/PI3K $\gamma$ signaling pathway (11). Tregs are a subset of CD4+ T cell and they play a key role in immunosuppression. It is recognized that the level of Tregs was significantly increased in tumor and peripheral blood of patients with cancer, and the function of immunosuppression was enhanced at the same time (12). However, the mechanism is unknown. Mrizak et al. (13) discovered that tumor exosomes can recruit Tregs in peripheral blood via CCL20. The exosomes also recruited other subtypes of $\mathrm{T}$ cells and then converted them into Tregs. After incubating with exosomes, Tregs significantly expanded and possessed stronger immunosuppressive function. In addition to tumor released exosomes, exosomes from tumor-associated macrophages can also transfer miRNAs that induced a Treg/Th17 cell imbalance to promote the development of ovarian cancer $(14,15)$. In all, exosomes can enhance tumor immunosuppression by affecting Tregs phenotype, properties and recruitment capacity. MDSCs are another important tumor immunosuppressive cells. Glioma-derived exosomes can enhance MDSC expansion and functional capacity via the miR-10a/Rora and miR-21/Pten Pathways (16).

Based on the role in tumor immunity discussed above, exosomes have potential application value. First, we can assess the immune status of patients with cancer. The specific nucleic acids and proteins carried by exosomes can realize quantitative detection. The results can be used for prognosis and predicting immunotherapeutic responses. Secondly, surface-bound proteins and contents of exosomes are derived from initial cells, so exosomes from tumor cells will be promising for tumor vaccine development. Rao et al. $(17,18)$ showed that DC-derived exosomes elicited tumor regression and similarly tumor-derived exosomes elicited tumor suppression in murine hepatocellular carcinoma models and humans in vitro. Third, exosomes are targets for immunotherapy. Besides, the bilayer membranes of exosomes are nanosized, which can protect exosomes from clearance. Therefore, exosomes can be used as drug delivery for tumor treatment or remodeling the immune microenvironment. However, there are still some problems to solve before clinical applicant. Based on our experience, there are still many impurities after extraction of exosomes though using ultracentrifugation. So, exploring a better purification method is in urgent need. Except proteins and RNA, the roles of lipids, DNA and metabolite carried by exosomes remain unclear. It is necessary to understand the exosome transport mechanism for drug delivery.

Exosomes are acting as important mediators of the crosstalk between tumor cells and the microenvironment and regulating tumor immunity. More rigorous researches need to be conducted in the future.

\section{Acknowledgements}

Funding: This study was supported by grants from the CAMS Innovation Fund for Medical Sciences (CIFMS) (No. 2016-I2M-1-001) and the National High-tech Research and Development Projects (863) (No. 2015AA020303).

\section{Footnote}

Conflicts of Interest: The authors have no conflicts of interest to declare.

\section{References}

1. Yoon SB, Chang JH. Extracellular vesicles in bile: a game changer in the diagnosis of indeterminate biliary stenoses? Hepatobiliary Surg Nutr 2017;6:408-10.

2. Ruivo CF, Adem B, Silva M, et al. The Biology of Cancer Exosomes: Insights and New Perspectives. Cancer Res 2017;77:6480-8. 
3. Hoshino A, Costa-Silva B, Shen TL, et al. Tumour exosome integrins determine organotropic metastasis. Nature 2015;527:329-35.

4. Moroishi T, Hayashi T, Pan WW, et al. The Hippo Pathway Kinases LATS1/2 Suppress Cancer Immunity. Cell 2016;167:1525-39.e17.

5. Hellwinkel JE, Redzic JS, Harland TA, et al. Gliomaderived extracellular vesicles selectively suppress immune responses. Neuro Oncol 2016;18:497-506.

6. Mirzaei R, Sarkar S, Dzikowski L, et al. Brain tumorinitiating cells export tenascin-C associated with exosomes to suppress T cell activity. Oncoimmunology 2018;7:e1478647.

7. Chen G, Huang AC, Zhang W, et al. Exosomal PD-L1 contributes to immunosuppression and is associated with anti-PD-1 response. Nature 2018;560:382-6.

8. Bévant K, Coulouarn C. Landscape of genomic alterations in hepatocellular carcinoma: current knowledge and perspectives for targeted therapies. Hepatobiliary Surg Nutr 2017;6:404-7.

9. Ning Y, Shen K, Wu Q, et al. Tumor exosomes block dendritic cells maturation to decrease the $\mathrm{T}$ cell immune response. Immunol Lett 2018;199:36-43.

10. Shen Y, Guo D, Weng L, et al. Tumor-derived exosomes educate dendritic cells to promote tumor metastasis via HSP72/HSP105-TLR2/TLR4 pathway. Oncoimmunology 2017;6:e1362527.

Cite this article as: Yang H, Sun L, Mao Y. The role of exosomes in tumor immunity. Ann Transl Med 2018;6(Suppl 2):S116. doi: 10.21037/atm.2018.12.03
11. Wang X, Luo G, Zhang K, et al. Hypoxic tumor-derived exosomal miR-301a mediates M2 macrophage polarization via PTEN/PI3K $\gamma$ to promote pancreatic cancer metastasis. Cancer Res 2018;78:4586-98.

12. Sun L, Xu G, Liao W, et al. Clinicopathologic and prognostic significance of regulatory $\mathrm{T}$ cells in patients with hepatocellular carcinoma: a meta-analysis. Oncotarget 2017;8:39658-72.

13. Mrizak D, Martin N, Barjon C, et al. Effect of nasopharyngeal carcinoma-derived exosomes on human regulatory T cells. J Natl Cancer Inst 2014;107:363.

14. Zhou J, Li X, Wu X, et al. Exosomes released from tumorassociated macrophages transfer miRNAs that induce a Treg/Th17 cell imbalance in epithelial ovarian cancer. Cancer Immunol Res 2018;6:1578-92.

15. Miura K. The day will come to treat HCC in a drugstore? Hepatobiliary Surg Nutr 2017;6:420-3.

16. Guo X, Qiu W, Liu Q, et al. Immunosuppressive effects of hypoxia-induced glioma exosomes through myeloidderived suppressor cells via the miR-10a/Rora and miR21/Pten Pathways. Oncogene 2018;37:4239-59.

17. Lu Z, Zuo B, Jing R, et al. Dendritic cell-derived exosomes elicit tumor regression in autochthonous hepatocellular carcinoma mouse models. J Hepatol 2017;67:739-48.

18. Rao Q, Zuo B, Lu Z, et al. Tumor-derived exosomes elicit tumor suppression in murine hepatocellular carcinoma models and humans in vitro. Hepatology 2016;64:456-72. 